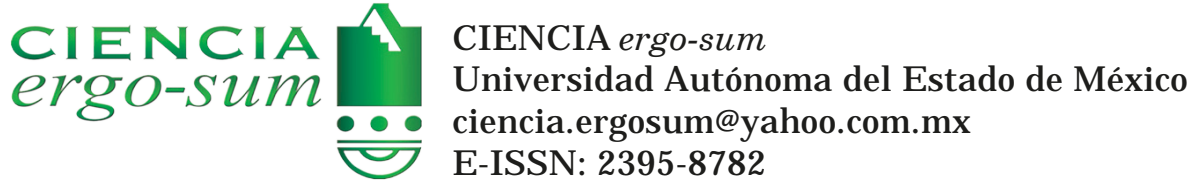

\title{
Special features of radio pulse spectral density analysis
}

Chubykalo, Andrew; Espinoza, Augusto; Kuligin, Victor

Special features of radio pulse spectral density analysis

CIENCIA ergo-sum, Número especial "Retos de la física no lineal" 2020| e103

Universidad Autónoma del Estado de México, México

Esta obra está bajo una Licencia Creative Commons Atribución-NoComercial-SinDerivar 4.0 Internacional .

Chubykalo, A., Espinoza, A. y Kuligin, V., (2020). Special features of radio pulse spectral density analysis. CIENCIA ergo-sum, Número especial "Retos de la física no lineal". https://doi.org/10.30878/ces.v27n4a2 


\title{
Special features of radio pulse spectral density analysis
}

\section{Características especiales del análisis de la densidad espectral de pulsos de radio}

\author{
Andrew Chubykalo \\ Universidad Autónoma de Zacatecas, México \\ achubykalo@yahoo.com.mx \\ (D) http://orcid.org/0000-0001-5324-1834 \\ Augusto Espinoza \\ Universidad Autónoma de Zacatecas, México \\ drespinozag@yahoo.com.mx \\ (1) http://orcid.org/0000-0002-1478-7946 \\ Victor Kuligin \\ Voronezh State University, Russia \\ victor_kuligin@mail.ru \\ (D) http://orcid.org/0000-0001-8859-8104
}

\author{
Recepción: 28 de mayo de 2019
}

Aprobación: 10 de febrero de 2020

\begin{abstract}
The spectrum analysis of the periodic sequence radio pulses is often described in textbooks. However, if this method is applied to short radio pulses with a large period between them, then large errors occur. In this article, we described a new method of pulse gating. This method allows us to measure the spectral density of radio signals with high duty cycle. The main advantages of our method are a high signal-to-noise ratio, a large dynamic range of measurements, and a higher accuracy of spectral density measurements.

KEYWORDS: Spectral line amplitudes, spectral density measurements, LF-amplifier.

\section{Resumen}

En los libros de texto se describe el análisis del espectro de la secuencia periódica de pulsos de radio. Sin embargo, si éste se aplica a pulsos de radio cortos con un periodo largo entre ellos, se producen grandes errores. En este artículo se describe el método de activación por pulso, el cual permite medir la densidad espectral de las señales de radio con un ciclo de trabajo alto. Entre las principales ventajas que posee está una alta relación señal/ruido, un amplio rango dinámico de mediciones y una mayor precisión de las mediciones de densidad espectral.
\end{abstract}

Palabras Clave: amplitudes de líneas espectrales, medición de densidad espectral, amplificador LF.

\section{INTRODUCTION}

The method of analyzing the spectrum of radio pulses was developed long ago (Kharkevich, 1960; Papoulis, 1977). There are two options for analysis. The first option involves measuring the amplitude of spectral lines. The second option is related to the measurement of the spectral density of a sequence of radio pulses.

In their form and in the structure of their circuit implementation, the spectral density analyzer, and the conventional panoramic spectrum analyzer (PSA) do not differ from each other. However, the principles of their work differ significantly. The article describes the special features of the analysis of spectral density of radio pulses, which is not paid attention in the literature. 
A typical block diagram of such analyzers is shown in Figure 1.

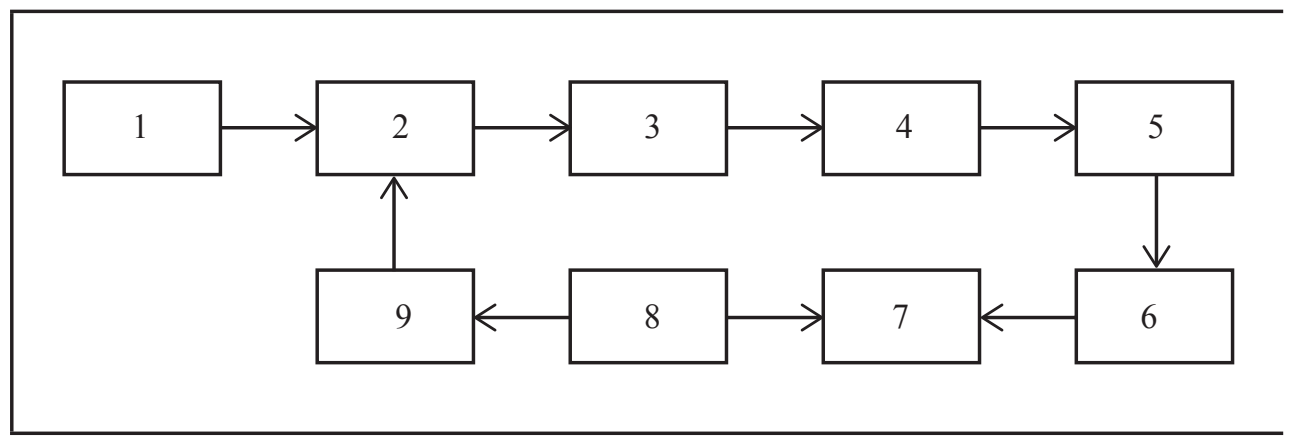

FIGURE 1

Simplified block diagram of a spectrum analyzer

Source: Own elaboration.

Note: 1-input device; 2-mixer; 3-Intermediate-frequency amplifier (IFA); 4-narrowband filter; 5-detector; 6-LF-amplifier;

7-cathode-ray tube (CRT); 8-horizontal scan generator; 9-heterodyne (H).

Although the principle of operation of a conventional spectrum analyzer is well known to specialists, we recall some points. The frequency of the local oscillator is tuned synchronously with the horizontal scanning of the CRT. When the spectral line arriving at the broadband IFA to the filter coincides with the frequency of the narrowband filter, a signal appears at the output of the filter. This signal is detected, amplified, and fed to vertically deflecting CRT plates. This method is resonant, because the energy of the spectral line is long enough accumulated in the resonant system of the filter.

To implement this method of analysis it is necessary:

a) The distance between the spectral lines must be greater than the filter bandwidth, else we do not differ two neighbor lines.

b) The filter adjustment time must be significantly longer than the time constant of the narrowband filter, [1] otherwise we will "slip" through the spectral line, that is, we will not see it.

When analyzing the spectral density of radio pulses, following with a bigh duty cycle, the first condition is not satisfied. The duty cycle is the ratio of the pulse repetition period $T$ to the duration of a single pulse $\tau$.

$$
S=T / \tau .
$$

The value of $S$ can reach several thousand. The reason for the difficulty of ordinary spectral analysis is that many spectral lines fall simultaneously into the filter bandwidth. As a result, in a narrowband filter there is no resonant accumulation of energy, which is typical of a conventional spectrum analyzer.

At the output of the narrowband filter, a transition process arises from the impact of a short pulse, which quickly fades long before the next pulse approaches. This reaction (the maximum response to a radio pulse) is proportional to the first approximation of the spectral density of this pulse.

In other words, the maximum value of the voltage response at the output of the narrowband filter is related to the energy passing through the filter passband. In Figure 2 it is shown an illustration of measuring the amplitude of a spectral line and measuring the spectral density. Now we need to get acquainted with the features of the spectral density analysis. 


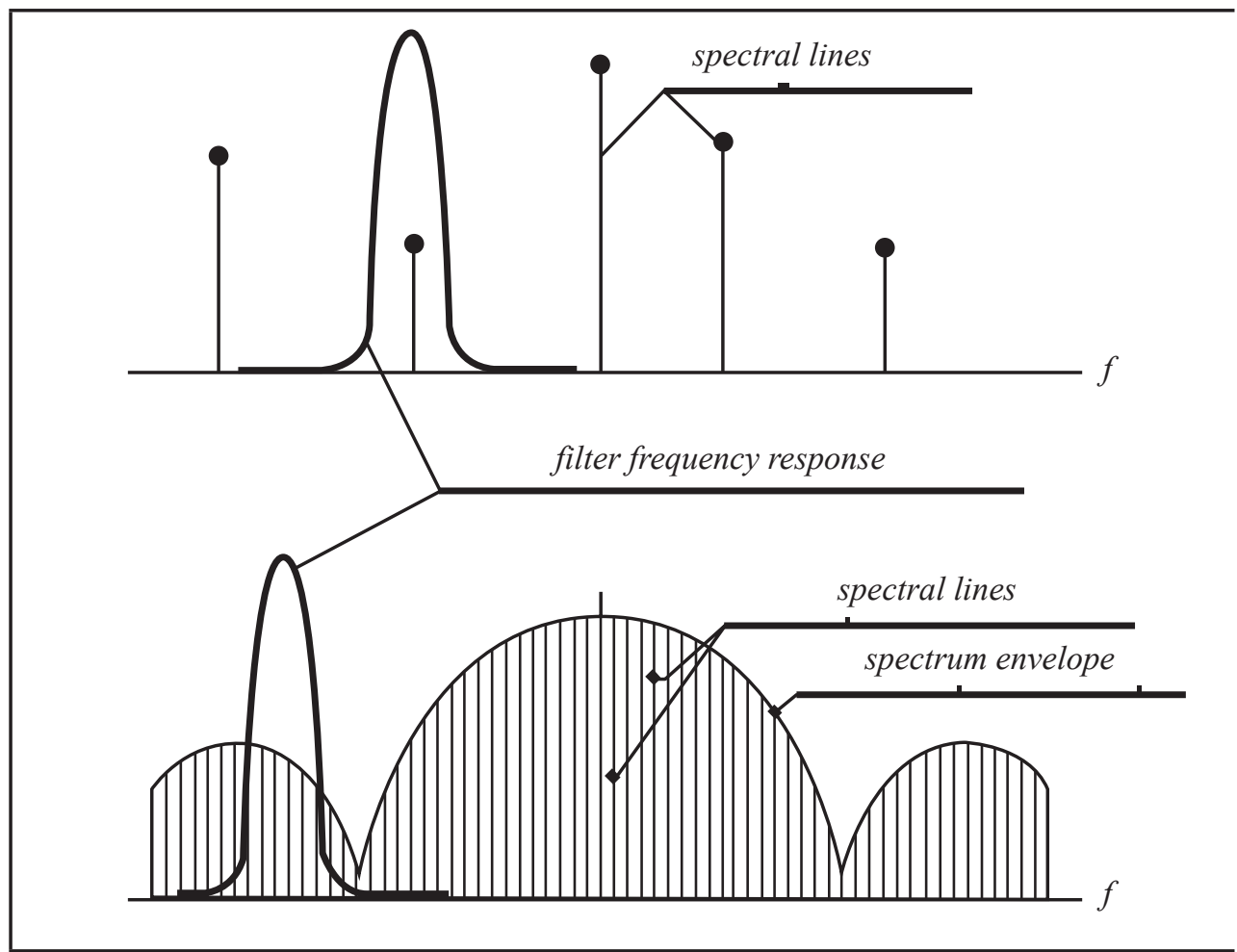

FIGURE 2

Measuring of the amplitude of a spectral line and of the spectral density Source: Own elaboration.

\section{Narrowband FiLter outPut transition Process}

When analyzing the spectral density, the absolute values of the spectral density are practically not measured. Researchers are usually interested in the frequency distribution of the relative magnitude of the spectral density. To avoid terminological confusion, we will denote by the symbol $S(i \omega)$ the complex spectral density of the pulse under study. The energy density of the spectrum will be proportional to the product of the complex conjugate values $S(i \omega) \cdot S^{*}(i \omega)$.

Consider a filter with a symmetric ("single-peaked") frequency response. The impulse response of such a filter (the filter's response to the effect of a signal in the form of a $\delta$-function) can be written as

$$
g(t)=A(t) e^{i \omega} f^{t}
$$

where $A(t)$ is the envelope (amplitude) of the oscillations at the filter output, $\omega_{f}$ is the oscillation frequency at the filter output. From the broadband IFA output (the bandwidth of which is wider than the spectrum of the pulse under study), a radio pulse is fed to the input of this narrow-band filter. At the output of the narrow-band filter, we get a signal

$$
u(t)=U(t) \cos \left[\omega_{0} t+\varphi(t)+\varphi_{0}\right]
$$

where $U(t)$ is the pulse envelope; ; $\omega_{0}$ - carrier frequency, $\varphi(t)$ is the phase change inside the pulse (AM or FM modulation), $\varphi_{0}$ - random initial phase of oscillation. The carrier frequency of the analyzed pulse varies slowly 
with time due to the tuning of the heterodyne frequency. In other words, the spectrum of a sequence of short pulses is slowly rearranged relative to the center frequency of the filter.

We consider very short pulses. The local oscillator tuning frequency is very low. We assume (this is the case in practice) that the change in frequency (due to the tuning of the local oscillator frequency) is very small during the duration of the pulse and does not affect the measurement of spectral density. We can assume that the oscillator frequency is constant while a short pulse is in effect. We will not consider issues related to the error estimation due to frequency tuning, asymmetry of the frequency response of the filter and due to other factors. Values: $A(t), U(t), \varphi(t)$ - we will consider slowly changing compared with the oscillation period of the carrier frequency of the signal or filter.

Using the Duhamel integral, we write the response at the filter output

$$
u_{\text {out }}(t)=h(0) u(t)+\int_{0}^{t} u(\tau) g(t-\tau) d \tau=\int_{0}^{t} u(\tau) g(t-\tau) d \tau,
$$

Since for a narrowband filter the value $h(0)=0$.

$$
u_{\text {out }}(t)=\operatorname{Re} \int_{0}^{t} u(\tau) A(t-\tau) e^{i \omega_{f}(t-\tau)} d \tau
$$

Now let us represent $A(t-\tau)$ as a Taylor series in powers of $\tau$.

$$
A(t-\tau)=\sum_{n=0}^{\infty} \frac{(-1)^{n}}{n !} \frac{d^{n} A(t)}{d t^{n}} \tau^{n}
$$

Using this result, we bring (2.1) to the following form

$$
u_{\text {out }}(t)=\operatorname{Re} e^{i \omega_{f} t} \sum_{n=0}^{\infty} \frac{(-1)^{n}}{n !} \frac{d^{n} A(t)}{d t^{n}} \int_{0}^{t} \tau^{n} u(\tau) e^{-i \omega_{f} \tau} d \tau
$$

We denote the integral as

$$
S\left(\omega_{f}, t\right)=\int_{0}^{t} u(\tau) e^{-i \omega_{f} \tau} d \tau
$$

This value represents the current spectral density of the filter response at the filter output. This function is time dependent and shows the process of spectrum formation. It is not difficult to show that

$$
\frac{d^{n}}{d \omega^{n}} S\left(\omega_{f}, t\right)=(-i)^{n} \frac{d^{n}}{d \omega_{f}^{n}} \int_{0}^{t} u(\tau) e^{-i \omega_{f} \tau} d \tau=(-i)^{n} \int_{0}^{t} u(\tau) \tau^{n} e^{-i \omega_{f} \tau} d \tau .
$$

We finally have

$$
u_{\text {out }}(t)=\operatorname{Re}\left[e^{i \omega_{f} t} \sum_{n=0}^{\infty} \frac{(-1)^{n}}{n !} \frac{d^{n} A(t)}{d t^{n}} \frac{d^{n} S\left(\omega_{f}, t\right)}{d \omega_{f}^{n}}\right]
$$


We obtained a result that will allow us to analyze the process of measuring the spectral density of the radio pulse under study.

\section{Formation OF INFORMATION ABOUT THE SPECTRAL DENSITY}

The transition process at the output of the filter can be divided into two parts. The first part is the process of forming the output response. The second part is a complete response to the impact of the pulse. This is due to the finite duration of the pulse being analyzed. The response envelope is shown in Figure 3.

We write the two parts of the response (2.3) separately:

$$
u_{\text {out } 1}(t)=\operatorname{Re} e^{i \omega_{f} t} \sum_{n=0}^{\infty} \frac{(-1)^{n}}{n !} \frac{d^{n} A(t)}{d t^{n}} \frac{d^{n} S\left(\omega_{f}, t\right)}{d \omega_{f}^{n}}, \quad\left(t<\tau_{0}\right),
$$

where $S\left(\omega_{f}, t\right)=\int_{0}^{t} u(\tau) e^{-i \omega_{f} \tau} d \tau$,

$$
u_{\text {out } 2}(t)=\operatorname{Re} e^{i \omega_{f} t} \sum_{n=0}^{\infty} \frac{(-1)^{n}}{n !} \frac{d^{n} A(t)}{d t^{n}} \frac{d^{n} S\left(\omega_{f}\right)}{d \omega_{f}^{n}}, \quad\left(t \geq \tau_{0}\right),
$$

where $S\left(\omega_{f}\right)=\int_{0}^{\tau_{0}} u(\tau) e^{-i \omega_{f} \tau} d \tau$. The quantity $S\left(\omega_{f}\right)$ is the desired spectral density. It is easy to see that information about the spectral density of the pulse $S\left(\omega_{f}\right)$ is contained in the second part of the transient process.

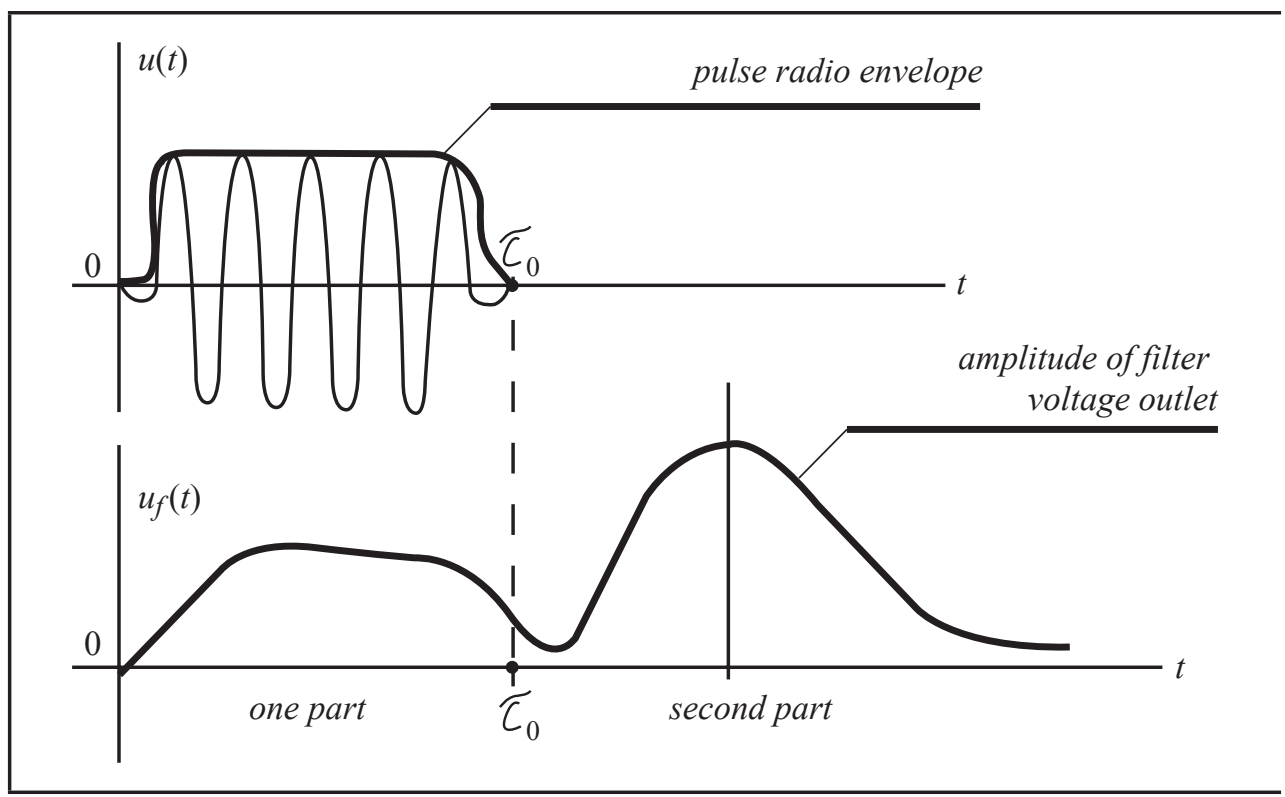

FIGURE 3

Two parts of the transition process

Source: Own elaboration.

In the process of analyzing the spectral density, the frequency response of the filter moves relative to the filter (Figure 4) due to the rearrangement of the heterodyne (local oscillator). Therefore, each response carries information about different parts of the spectral density. 


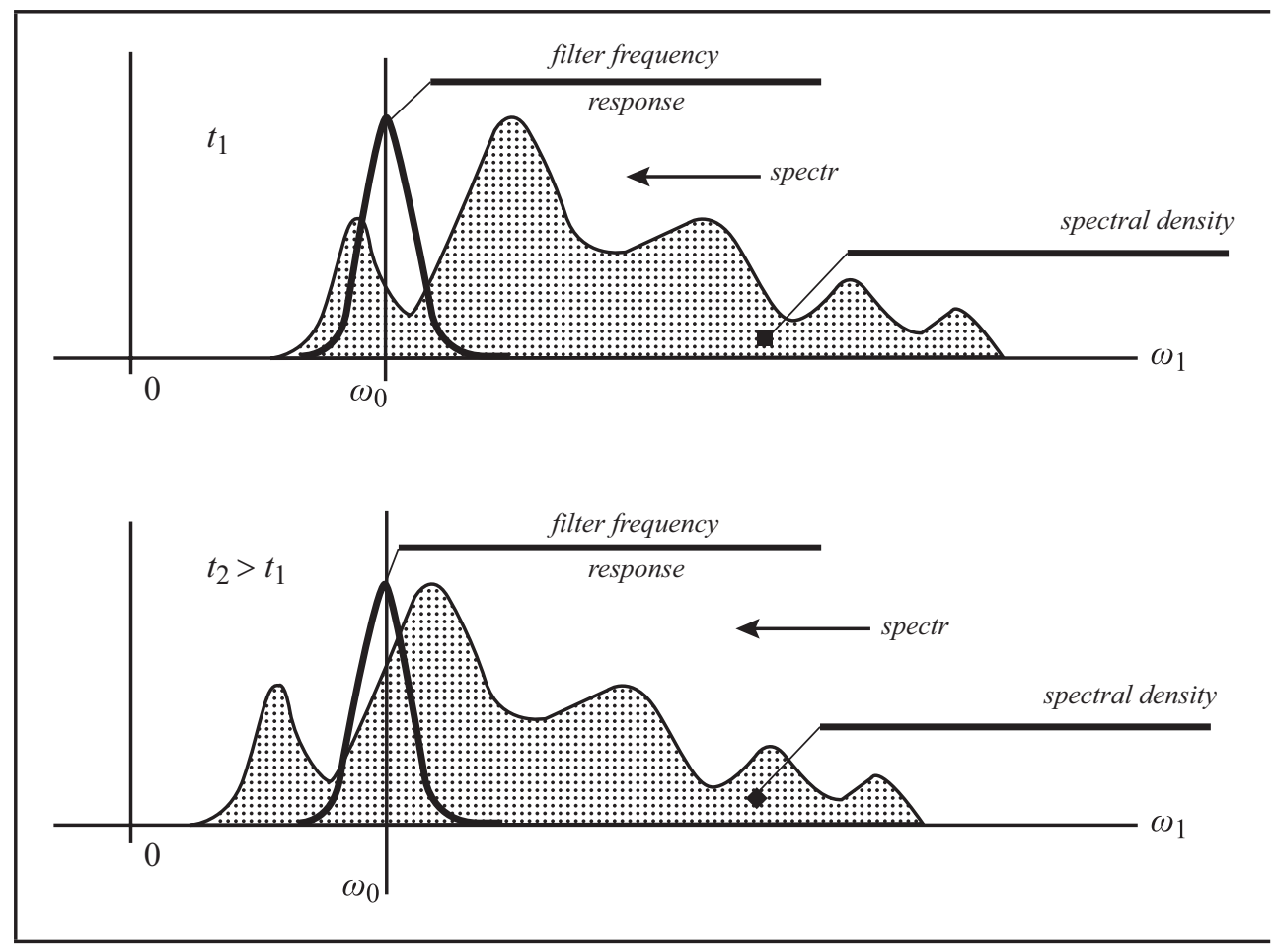

FIGURE 4

Movement of the spectral density of the pulse relative to the frequency response of the filter due to the frequency tuning of the local oscillator (heterodyne)

Source: Own elaboration.

Now it is necessary to determine the pulse duration, since the "tail" of the pulse under study can be delayed in time. We will determine its duration based on the required accuracy of spectral density measurement. For example, we can consider the duration of a pulse to be such a time interval, which contains 99.9 percent of energy, and the share of the "tail" is 0.1 percent.

We now consider the second part of the transition process (3.2). Information about the value of the spectral density contains only the first member of the series. The remaining members will cause a measurement error.

\section{TyPiCAL SPECTRAL DENSITY MEASUREMENT ERRORS}

Now we need to evaluate the contribution of the members of the power series in expression (3.1). The impulse response $A(t)$ we can write through the complex transfer coefficient of the filter $k(i \Omega)$

$$
A(t)=\int_{-\infty}^{\infty} k(i \Omega) e^{i \Omega t} d \Omega
$$

where $\Omega$ is the detuning relative to the center frequency of the filter. Using this relationship, it can be shown that

$$
\left|\frac{d^{n} A(t)}{d t^{n}}\right| \leq \frac{2}{(n+1) !}\left(\frac{\Delta \omega_{f}}{2}\right)^{n+1},
$$


where $\Delta \omega_{f}$ is the filter bandwidth. Similarly, it can be shown that

$$
\left|\frac{d^{n} S\left(i \omega_{f}\right)}{d \omega_{f}^{n}}\right| \leq S\left(i \omega_{f}\right)\left(\tau_{0}\right)^{n+1},
$$

where the maximum value of the spectral density is taken. The order of magnitude of the $\mathrm{n}$-th term of the series (3.1) with respect to the first is

$$
\delta_{n}=2 \frac{\left(\Delta \omega_{f} \tau_{0}\right)^{n+1}}{2 n(n+1) !}
$$

Therefore, the better the condition $\Delta \omega_{f} \tau_{0}<\pi$, the faster the series converges and the more accurately the spectrum is measured. It is also necessary to pay attention to the following circumstance. The peculiarity of the measurement technique is that the spectral density value is read from the CRT screen by the maximum value of the magnitude of the response to the pulse. Changing the frequency of the local oscillator (heterodyne) leads to the fact that the spectral density slowly moves relative to the resonant frequency of the filter. At the moment, when a pulse arrives, a response is formed at the filter output. Due to the slowness of tuning the heterodyne on the CRT screen, this response is displayed in the form of a "stick"-vertical line. We take the amplitude of this line as the value of the spectral density.

With a narrow bandwidth, we measure the correct spectral density values where it is large. At those points where the spectral density is small, there is a "masking" of zeros. From Figure 3 that the measurement error can occur when the maximum value of the first part of the transient process exceeds the maximum value of the response of the second part of the process. In this case, we cannot measure the actual value of the spectral density. The second factor causing the appearance of an error is the influence of the second and other members of the series, when the value of the first term (carrying information about the spectral density) is small compared to them.

The Figure 5 shows the process of generating responses when a rectangular pulse is applied to a dual-circuit narrowband filter. In the plots, we can observe that the first part of the transition process "masks" information about the spectral density in those areas where the spectral density envelope is close to zero. The rate of change of frequency is small compared with the duration of the signal at the output of the filter. Therefore, the signal on the screen of the tube will be similar to a narrow pulse. The envelope of the spectrum almost coincides with the tops of the pulses on the screen. If we analyze the density of the spectrum of a rectangular radio pulse, then on the CRT screen we will see not the spectrum envelope, which is a module of the function $\sin \omega \tau / \omega \tau$, but something different from it (the graph on a dark background of Figure 5 (right side)). "Zeros of spectral density" masked by parts of the transition process.

These errors are illustrated in Figure 6, where a typical CRT screen is shown.

Remark. In the general case, spectral density measurement is associated with the solution of two interdependent problems

1. First, the fight against the masking action of the first part of the transition process and spurious masking "bursts" in the second part of the transition process.

2. Secondly, the struggle to increase the dynamic range of the analysis with respect to the noise level of the spectral density analyzer.

It is also necessary to eliminate measurement errors that arise due to the influence of the second and other members of the series (3.3) and noise, which can add their "contribution" to the measurement results. 


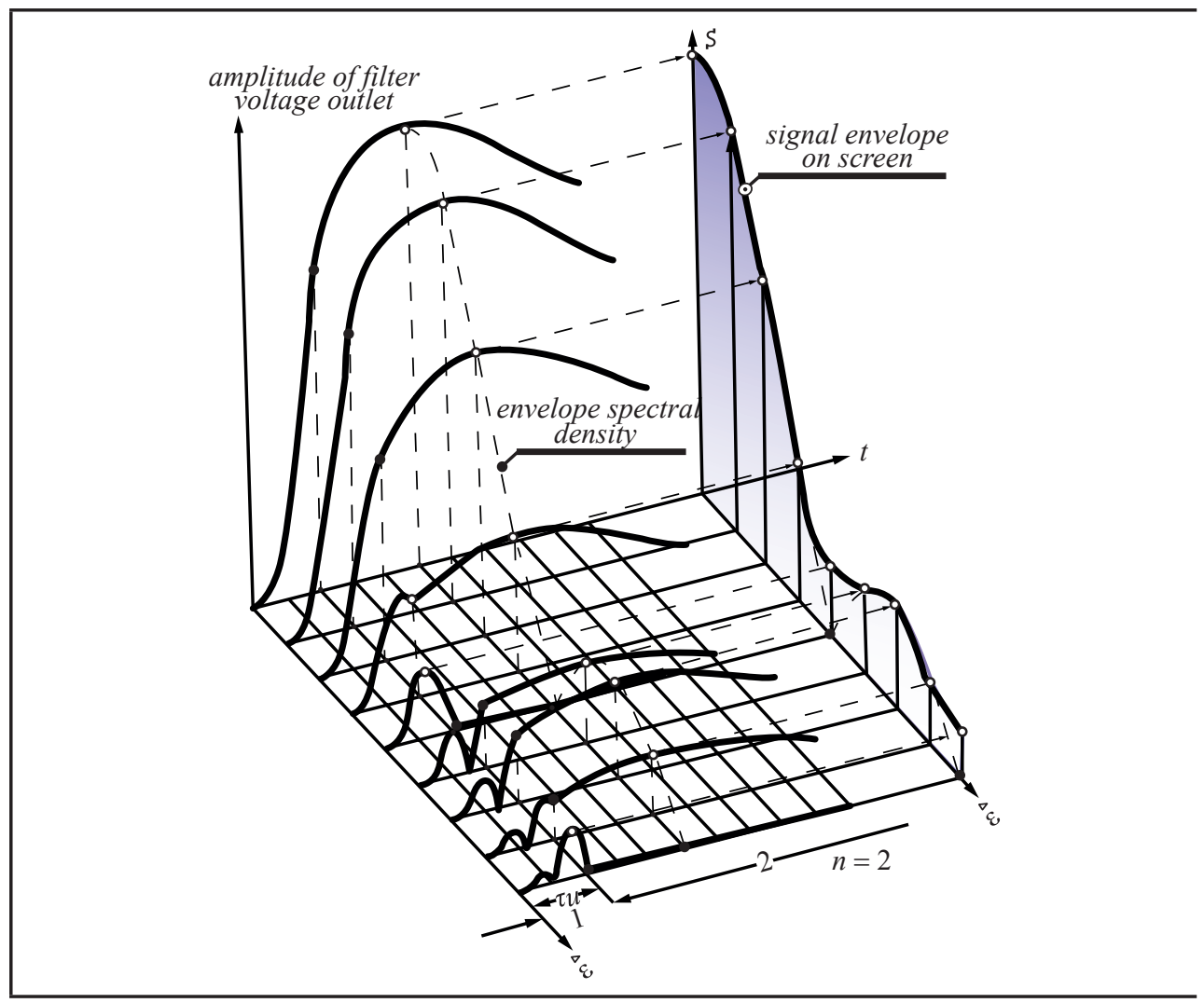

FIGURE 5

Transients at the output of the dual-circuit filter when exposed to a rectangular radio pulse Source: Own elaboration.

Note: The background color is the reproduction of the spectral density on the CRT screen. The effect of "masking" the spectrum in the area of zeros is visible. The dashed line in the center, passing through the light points (maximums of responses) is the true spectral density envelope.

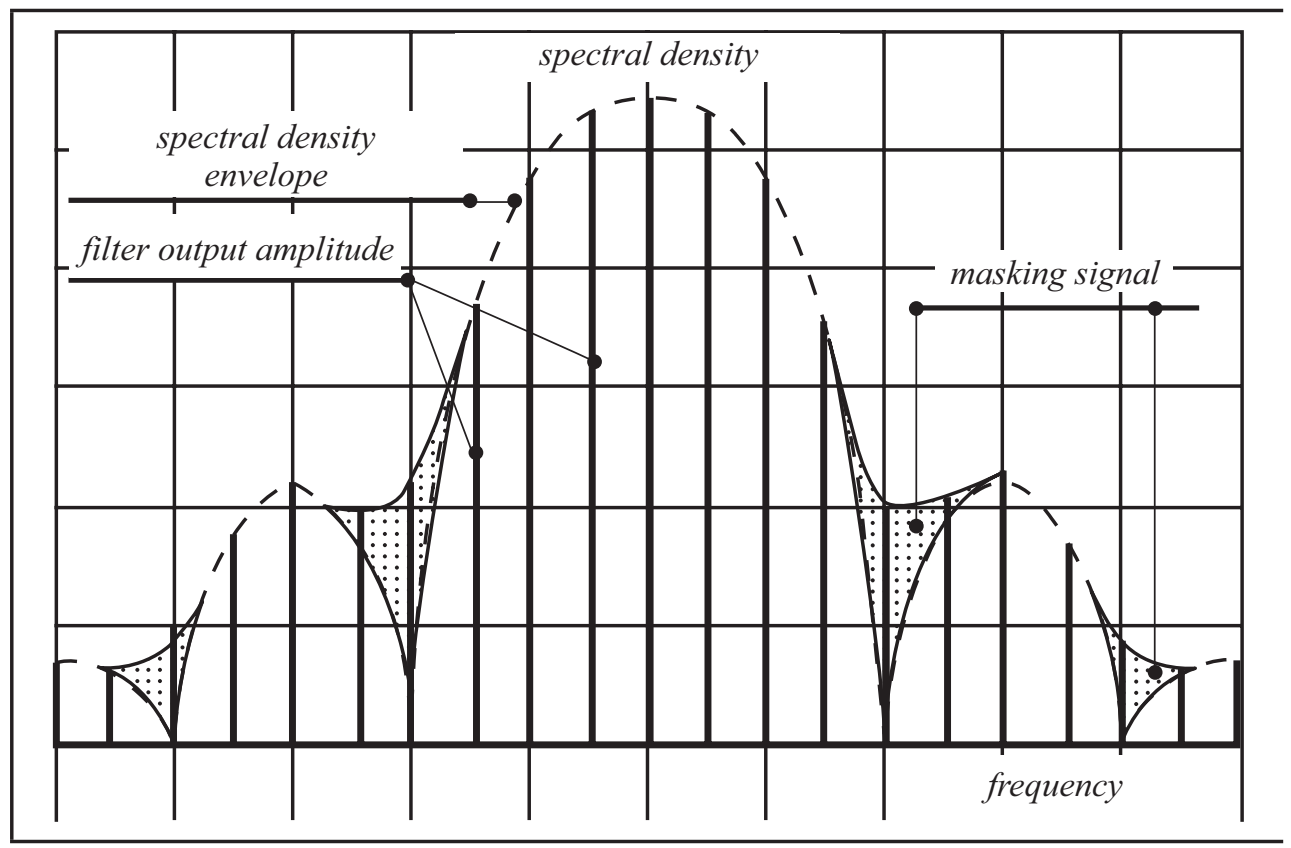

FIGURE 6

Distortion of "zeros" in the analysis of spectral density

Source: Own elaboration. 


\section{GATING AS A METHOD TO IMPROVE MEASUREMENT ACCURACY}

It is obvious that the narrower the bandwidth of the filter, the more accurately we can measure the spectral density of a sequence of pulses of high duty cycle. This can be seen from the estimate of the convergence of the series (3.3). However, there is a serious limitation. Reducing the filter bandwidth automatically reduces the signal-to-noise ratio. This is because the energy level of the noise decreases inversely proportional to the width of the passband, and the energy of the spectral density is proportional to the square of the filter's bandwidth.

Recalculate the required values to the input of the spectral density analyzer. Let the level of the permissible power of the input microwave pulse is $40 \mathrm{~dB} / \mathrm{MW}$. Exceeding this power leads to non-linear distortions and a violation of the linearity of the dynamic characteristic. We will consider the dynamic range of a conventional spectrum analyzer with a monochromatic signal equal to $80 \mathrm{db}$. The power of the maximum response value $P_{\text {out }}$ Pout decreases in proportion to the square of the filter bandwidth. The noise power $P_{n}$ decreases in proportion to the bandwidth, and the power of the error signal $P_{\text {err }}$ is proportional to the fourth power (Figure 7 ).

If we consider the optimal condition when the error signal ("masking" zeros) is commensurate with the noise level, then the dynamic range will be about $30 \mathrm{~dB}$ (line $a-a$ in Figure 7). We carried out an approximate calculation for a dynamic range of $80 \mathrm{db}$. With a dynamic range of $60 \mathrm{~dB}$, the numbers will be "more modest". Standard dynamic range will be about $20 \mathrm{~dB}$. This range can be increased up to $35-40 \mathrm{~dB}$ (depending on the dynamic range on a monochromatic signal), applying the signal gating at the filter output and a special frequency response of the filter, i.e., shift line $a-a$ to position $b-b$ and on.

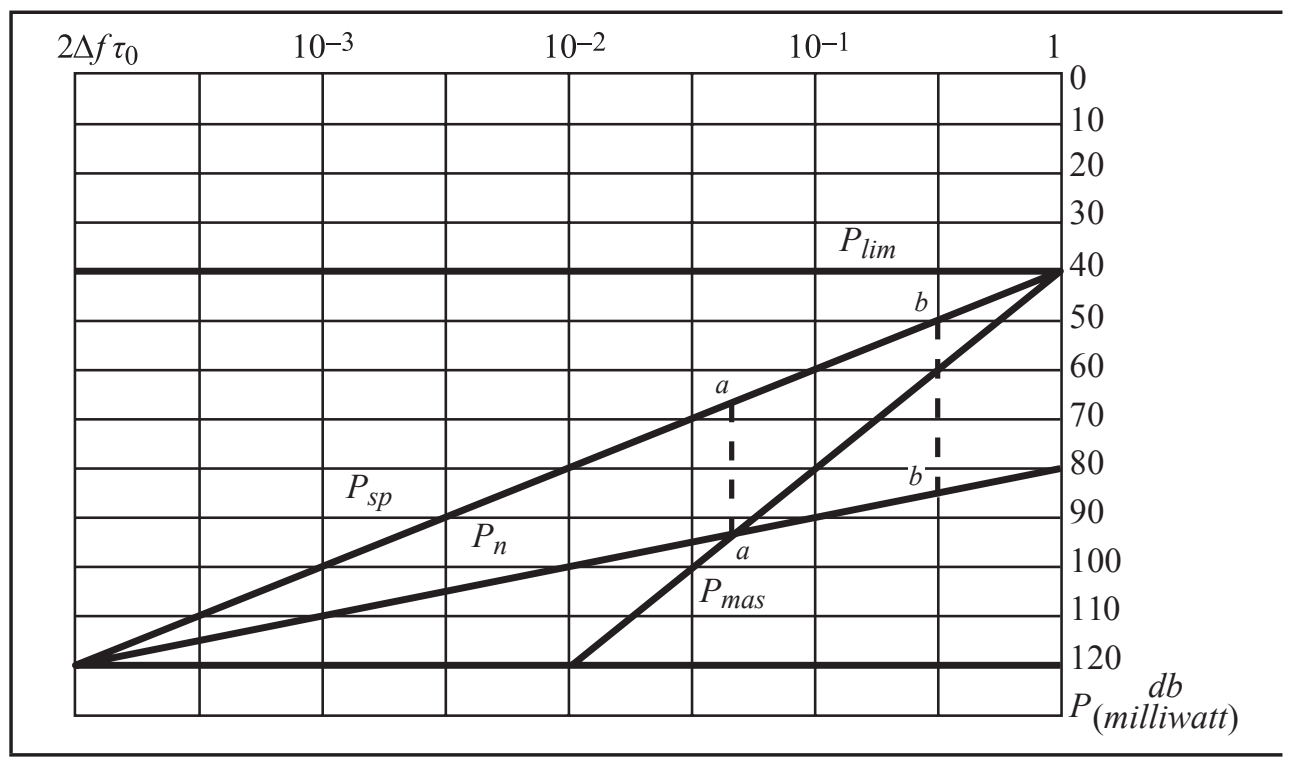

FIGURE 7

Dependence of the different spectral density powers on the filter bandwidth Source: Own elaboration.

Note: $P_{s p}$ is the maximum power of the spectrum at the filter output; $P_{l i m}$ is the permissible power at the input of the analyzer; $P_{n}$ - noise power; $P_{\max }$ - the maximum power of the error signal. All powers are recalculated to the input of the spectral density analyzer.

Consider the gating process. Figure 8 shows the first two components that form the response in the area where the spectral density information is "masked".

Information about the spectral density of AS (Figure 8) is contained in the first term of the expansion in the Taylor series (level 1). However, the second term of the expansion can sometimes be larger in amplitude. In this case, it "masks" the information and introduces distortions in the measurements (level 2). 


\section{The USE OF Gating WILL REDUCE THE ERROR}

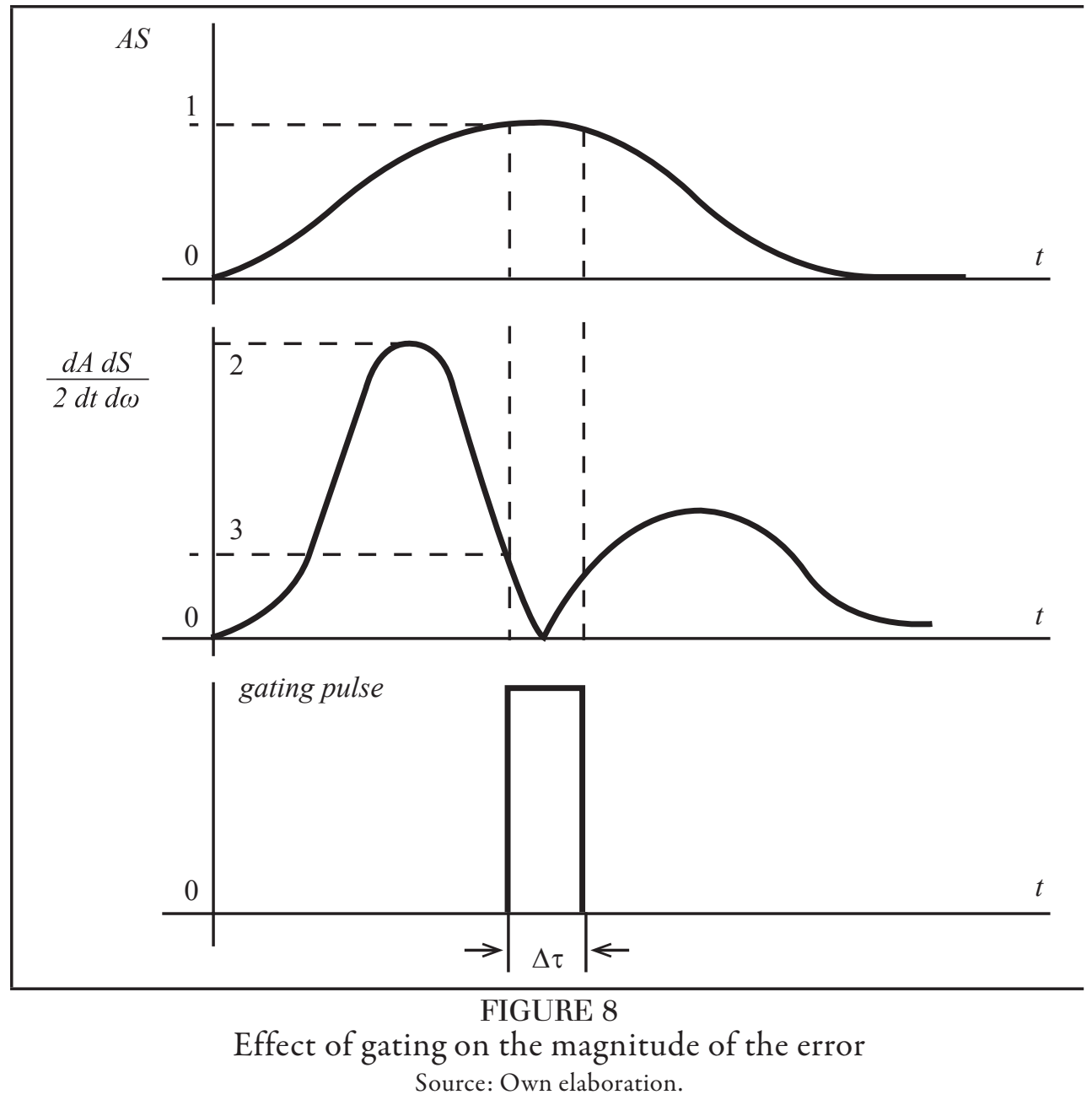

Gating is performed in the region where $A(t)$ is the maximum value. Here the first derivative of $A(t)$ passes through zero. Therefore, the "contribution" that the first derivative could give is substantially less. When gating will be significantly reduced area of masking information. The positive effect depends on the width of the strobe signal. The narrower the strobe, the better the result. Ultimately, we can bring the product $\Delta \omega_{f} \tau_{0}$ to $2 \pi$, providing a mode close to the optimal receiver mode.

But here we are waiting for two difficulties:

1. First, a narrow strobe leads to a decrease in signal energy after gating.

2. Secondly, an increase in $\Delta \omega_{f} \tau_{0}$ will lead to the fact that one should consider the error due to the contribution to the response of the higher terms of the series (3.1).

Therefore, the next logically justified step to increase the dynamic range and increase the measurement accuracy can be the use of a filter with a special frequency response. The idea is to use a filter whose pulse response envelope would have a "flat part" $\left(t_{1}-t_{2}\right)$, as shown in Figure 9. In this case, within the flat part of the impulse response $\left(t_{1}-t_{2}\right)$, all derivatives $\frac{d^{n} A(t)}{d t^{n}} \frac{d^{n} S\left(i \omega_{f}\right)}{d \omega_{f}^{n}}$ will be equal to zero for all $n>0$. This will increase the strobe width, and will allow to furtherexpand the filterbandwidth, i.e., increase the dynamic range of measurements in relation to the noise level. The frequency response of such a filter $k\left(i \omega_{f}\right) k(i \omega)$ should look like $\left|\sin \left(\omega-\omega_{f}\right) \tau /\left(\omega-\omega_{f}\right) \tau\right|$, as shown in Figure 9. 


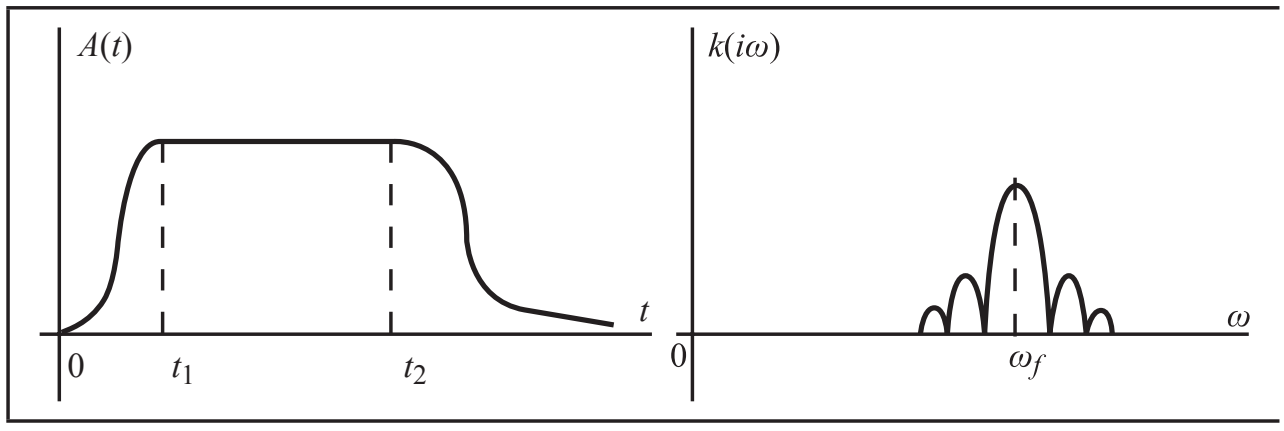

FIGURE 9

Pulse response with flat part

Source: Own elaboration.

\section{Practical test of the method}

The proposed method of improving the accuracy of measurements and increasing the dynamic range does not require significant changes in the modern panoramic spectrum analyzer. A conventional device is supplemented with a special filter and a gating circuit at the output of the filter. The block diagram of such an analyzer is shown in Figure 10.

The video signal from the incoming radio pulse is output from the mixer. The signal is amplified by the block 10. A start pulse is generated from it, which is fed to the strobe generation device 11 with a time delay. At 12, the filter response gating process takes place. This response is amplified by a narrowband amplifier 13, where its "expansion" occurs. Block 14 generates an additional signal for gating an electron beam (increase in beam current). Electronic gating ("backlight" or increase the brightness of the electronic image on the screen of a CRT) is carried out at the time when the response from the amplifier 6 reaches its maximum value. Thus, we disable reproduction of parts of the transition process that are unnecessary for analyzing the spectrum.

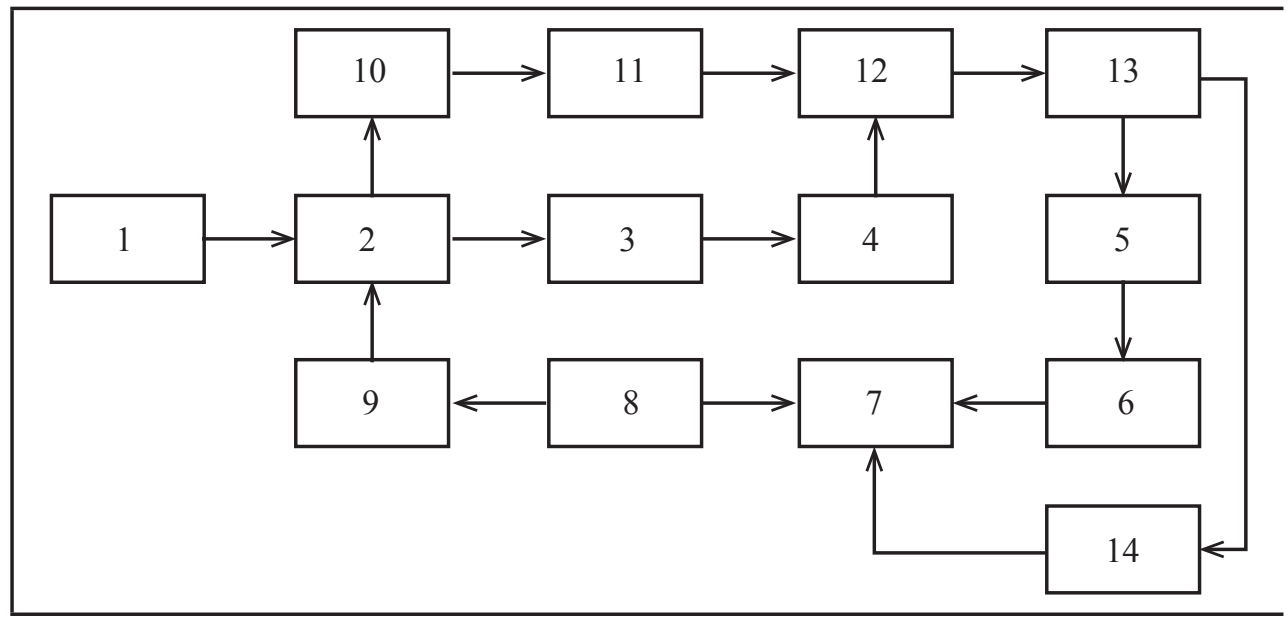

FIGURE 10

The block diagram of the spectral density analyzer with gating

Source: Own elaboration.

Note: 1-input device; 2-mixer; 3-broadband UAC; 4-special filter; 5-detector; 6-bass amplifier; 7-cathode-ray tube CRT; 8horizontal scan generator; 9 is a local oscillator; 10-synchro-pulse shaper; 11-device for the formation of the gate with a delay in time;

12-gating device; 13-HF amplifier and pulse expander; 14 is a pulse shaping device for illuminating a CRT beam. 
Let us dwell on the experimental verification of the gating method. The installation (corresponding to the block diagram shown in Figure 10) was built based on a panoramic spectrum analyzer. A standard instrument filter was used (the filter with the flat part of the impulse response was not used). Below are two photographs for illustration (Figure 11), which need to be explained.

Since the vertical "sticks" reproduced on the CRT screen are responses, not spectral lines, the photographs were taken in the "out of sync" mode between the pulse repetition rate and the instrument sweep frequency. In normal mode, the "sticks" ran across the screen, forming a solid picture.

In the gating mode, only the tops of the maxima of transients ("sticks") were highlighted. As a result of prolonged exposure to photographs, the maximum points, running across the CRT screen, formed a solid line denoting the spectral density envelope. A periodic sequence of pulses was generated by a reflective klystron ( $1 \mu$ sec duration and a duty cycle of 500). The generated pulse had a shape close to rectangular, and the frequency modulation, the value of which was controlled and could vary within certain limits.

In the two photographs below, the effect of "masking" the real spectrum by elements of transients is very clearly seen.

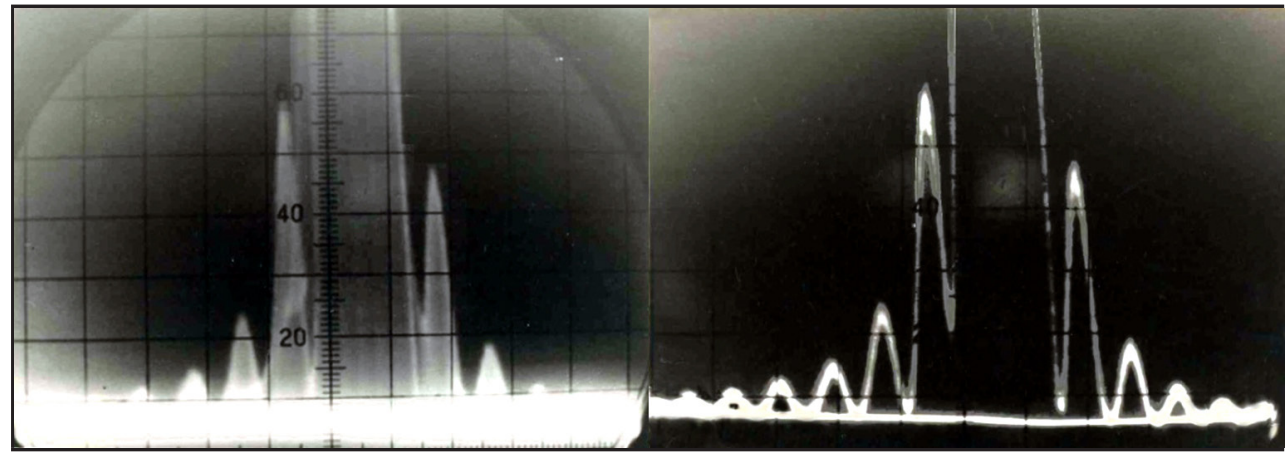

FIGURE 11

Effect of "masking" the real spectrum by elements of transients

Source: Own elaboration.

\section{Conclusion}

Therefore, as it was shown, the use of gating allows you to increase the measurement accuracy and dynamic range of the analysis. However, the measurement of the modulus of the spectral density does not allow to obtain complete information about the analyzed signal. It is necessary to obtain the phase-frequency characteristic of the spectral density.

Analysis of the measurement process allowed us to develop a method of signal gating at the output of the filter. The smallest measurement error is obtained if the gating moment is separated from the moment of arrival of the pulse by the value of the pulse duration.

Gating allows, without loss of accuracy, to increase the filter bandwidth to the optimum value and to increase the dynamic range of measurement by $20-30$ decibels.

Theoretical studies and experiments, [5], suggest that, thanks to small improvements in flowchart 9 (Dunsmore, 2012; Raju, 2008), it is possible to measure the phase response simultaneously with the measurement of the modulus of the spectral density. Moreover, it is possible to carry out measurements of a periodic sequence of high-frequency radio pulses, in which the initial phase of the carrier frequency is of a random nature. This question requires self-presentation.

Estimates show that such measurements of complex spectral density (Popovi'c Z, \& Kuester, 2013, considering the use of modern digital processing, will make it possible to describe a radio pulse in real time with an error not exceeding 1 to 5 percent. And this is already significant. 


\section{Prospective analysis}

Currently, panoramic spectrum analyzers (SPA) are used to measure the spectrum of monochromatic signals. The structure and principle of the SPA allows them to be used for the analysis of spectral density.

As already mentioned, the use of the SPA for this purpose does not allow for the high accuracy and dynamic range that occur when analyzing the spectrum of monochromatic signals.

The use of gating in the SPA will bring the dynamic range and accuracy of the SPA when analyzing the spectral density to the dynamic range and accuracy of the SPA when analyzing monochromatic signals.

The developed theory of spectral density measurement allows you to search for ways to measure not only the spectral envelope of a sequence of periodic pulses, but also to start searching for ways to realize the possibility of measuring the phase characteristics of spectral density.

This will allow, using measurement results, to calculate on a computer and describe the complete structure of the pulse in real time.

The use of gating can improve the performance of panoramic spectrum analyzers (SPA), which are used.

\section{REFERENCES}

Dunsmore, J. (2012). Handbook of microwave component measurements: With advanced VNA techniques. John Wiley \& Sons, Ltd.

Kharkevich, A. (1960). Spectra and analysis. Consultants Bureau.

Papoulis, A. (1977). Signal analysis. New York: McGraw-Hill.

Popovi'c, Z., \& Kuester, E. (2013). Principles of RF and microwave measurements. Colorado: University of Colorado Boulder, Colorado.

Raju, G. (2008). Microwave Engineering. I. K. International Publishing House Pvt. Limited.

\section{Notes}

[1] The filter time constant is the response time at the filter output when a signal from a "stepwise" type envelope is applied (the Heaviside function: it is 0 if $t$ is less than 0 , and it is 1 if $t>0$ ).

\section{BY-NC-ND}

